\title{
On the Reporting Characteristics of Chinese Infotainment App: A Case Study of Tanzi App \\ Mei Xie ${ }^{1, a, *}$, Qianru Wei ${ }^{2, b}$
}

${ }^{1}$ Mei Xie, professor of Political Science and Public Administration, University of Electronic Science and Technology of China, Chengdu, China

${ }^{2}$ Qianru Wei, graduate student of Political Science and Public Administration, University of Electronic Science and Technology of China, Chengdu, China

axiemei001@163.com, b36407548@qq.com

* Qianru Wei

Keywords: Tanzi app; reporting characteristics; audience; entertainment news

\begin{abstract}
Infotainment rose in the late 1980s and early 1990s and boomed at the end of the twentieth century via Internet and multimedia. Now, with the rapid economic development, the audience wants to get more amusement through news media after work, so plenty of infotainment sites and apps have sprung up, maximizing the entertainment function of the mass media, such as Buzzfeed, Vice, Weizeit, Happyjuzi, Tanzi and so on. This paper analyzes the current situation, reporting characteristics and the existing problems of Tanzi, an infotainment app from Chengdu, to make suggestions on how the infotainment app plays edutainment function of news media.
\end{abstract}

\section{The Proposal of Question}

British philosopher Herbert Spencer has suggested that the first task mankind needs to complete is to maintain and multiply life, and then if there is still energy, that is for entertainment. With the rapid development of productive forces, cultural and entertainment industry is increasingly prosperous. Whether in the study of communication, or in the news practice, the entertainment function of mass media becomes more and more prominent, and the demand for entertainment news is greater. On December 5, 2014, a new social media, Tanzi app, by Chengdu Economic Daily officially launched. Producing and sharing viral content as its core, Tanzi is to aggregate the most interesting, useful and lovely contents from a large number of social networks and to dig out local information and news, with five columns: "the Greatest Hits”, "Video", "Entertainment”, "Big list", and "Tanzifeed", including various topics, like star, film, world, people, history, skill, etc.. Each column has distinct orientation to provide plenty of high-quality infotainment. However, the aggregating development of entertainment news by infotainment app has brought in many problems. How to understand the essential attributes of entertainment news, how to standardize entertainment reports, and how to make entertainment news fit the audience's spiritual and cultural needs, are problems we need to explore.

\section{The Reporting Characteristics of Tanzi App}

In order to analyze the reporting characteristics of Tanzi, this paper randomly selects 736 pieces of news in "Video", "Entertainment", "Big List” and "Tanzifeed” from March 1, 2017 to May 31, 2017 as the sample. By analyzing the content, the author finds that Tanzi app has precise positioning to its target audience: the fans of celebrity and young people and pushes the articles with following characteristics:

\subsection{Keep up with hot entertainment topics and guide the audience with positive review}

As a kind of “economy of attention”, media industry must focus on its content. The report of tanzi app keeps up with the hot entertainment events, firmly complies with the principle of 
"forestalling", and breaks away from copying the large number of existing reports and scalable news production. It takes the original commentary as a selling point, with novel and unique perspective, so that the audience can get inspiration and sentiment during the entertainment, such as Ode to Joy II. Since it started to play on May 11, 2017, the concern about it has been growing from the plot to the characters' wearing style, the protagonist's view about love and career, and also the plot related to "buying house", "virgin complex" and other social problems. These all caused extensive discussion. Tanzi app can follow up the focus of the audience. "What is More Terrible than the Virgin Complex is how Qiu Yingying seeing the world", an article from the view of choosing partners criticizes different chastity views on man and woman in modern society, which encourages women who desire for independence but are often swayed by considerations of gain and loss, to be self-consistent, capable and independent. Another example is the Sichuan TV original music story show. After the broadcast, Tanzi published a review about Cui Jian's thirty-year rock career, who criticizes the fake singing and insists on the real performance, showing his original intention and devotion to music. Most reports of Tanzi can identify the proper perspective and guide the audience positively through entertaining and lucid way.

\subsection{Not act as the mouthpiece of stars and dare to criticize the evil phenomenon in entertainment circle}

On October 15, 2014, during the Symposium on Literary and Artistic Works in Beijing, President Xi Jinping criticized the current bad phenomenon in literary and artistic circles in China, such as unduly commercialization, heavy plagiarism, monotony, decrease of professional ethics and bad reputation of some artistic workers who go after the audience rating, cooperating with the stars to hype. Although Tanzi app is infotainment-oriented, it pays attention to the power of public figure, do not deliberately amplify the star halo and can focus on their hard work and efforts to establish a positive image for the audience, for example, in "He is the oldest actor in In the Name of People, but performed the highest level!”, the article highlights the actor Bai Zhidi who plays Chen Yanshi in In the Name of People. He is now in his seventies, but the performance is first-class, though rarely starred in the past few decades, yet shaped many vivid supporting characters. He takes his work as his foundation and give away the best spiritual thing to the audience. Meanwhile, for the hype by scandal, making gimmicks, "selling character setting" and other negative phenomenon, Tanzi app can maintain a correct attitude, speak with facts, improve the audience's critical consciousness and awareness, and expose the essence that stars idealized their screen image to cater to the audience preferences, and even mislead the audience. Tanzi calls on the stars to enhance influence through film and television works, and put the creation of outstanding works as the center, lest the audience astray.

\subsection{Focus on digging Chengdu elements, with local cultural appeal}

As a local infotainment new media, Tanzi is good at detecting Chengdu elements from daily life to fit the cultural needs of local audiences, like tell Chengdu people's own entertainment story. Also, Tanzi opened up the column "Big List" as "Chengdu special edition" to push original articles about Chengdu people regularly, Chengdu life guide, presenting decades of commercial development, cultural landscape, urban construction and other changes of Chengdu, vividly showing a different kind of Chengdu life and the unique charm of the city to lead the audience experience the down to earth life of Chengdu. "Knowing Chengdu people from his/her courtyard", "Since time flies...Let's crowdfund the memory of the city", "Listen to the courtyard story of Chengdu with Yuan Tingdong and Wang Hai", etc. all these articles describe the courtyard life of Chengdu, which is the projection of Chengdu leisure life and now is fading with the development of the city. Tanzi tries to evoke the collective memory of Chengdu people with the original articles of "disappearing courtyard" series and make the audience to understand and appreciate the beauty of traditional Chinese architecture, to explore the ancient courtyard culture of Chengdu. The content of this column is not only entertaining and popular, but also ideological and elegant with artistic knowledge, and emphasizes cultural connotation and spiritual taste, embodies the spirit of Chinese culture, reflecting the aesthetic pursuit of Chinese people. 


\subsection{Entertaining short video with streamlined content, lively form and creation}

As the brand section of Tanzi, "tanzikoushuiji" daily launches one or two pieces of about-four-minute funny short video which is the interpretation of re-processing film and TV works, popular songs, novel experience and wired event by video collage or live action shot with voiceover of dialect and catchword. It is humorous and close to the ordinary people's life, increasing the engaged audience. Movie Interpretation is a fixed section of Tanzi app. The selection of the film is rich, like Nerve, The Devotion of Suspect X and other popular commercial films, Nô Otoko and Split as the representative feature films. With highly concentrated information and creative points, Movie Interpretation abandons the lengthy bedding presentation and summarizes the film plot in a short time, so that the audience can be quickly into the film, fully mobilizing their emotions and causing resonance. In addition to the visual-effect-pursuing and entertaining short videos, Tanzi also introduced a series of short video named City Savage to record life and reality. "Strive for Life: They Can Still Play Volleyball without legs", "I thought he was a big brother, But He Wanted to Be Stephen Chow" and other inspirational short videos unfolds the ordinary people's life of fighting for their dreams and careers. These videos are encouraging, conveying the optimistic attitude towards life and making the audience contemplate on life.

\section{The Existing Problems and Thinking of Tanzi Report}

\subsection{News headlines should be rigorous, abandoning the large-scale network language or vulgar language}

Infotainment focuses on the eyeball effect. However, employing the transgressing and indecent words to describe entertainment stars and events, blindly chasing the sensory stimulation, and taking it as a gimmick to attract the audience eye, to meet the audience poor taste, all these are harmful to the long-term development of entertainment news and the cultivation of the audience personal taste. There is "title party" and "edge ball” phenomenon in information push. By using plenty of "catfight”, “dumbstruck", "lick the screen” and other network languages, selecting the most cheesy point of the news as the title to attract the audience to click, such as "Boys here are all dreaming to be pimp to sell the girls over the country to the United States as sex slave...”, the article with this title reveals human trafficking and forced prostitution in Tlaxcala state, Mexico, and that is normalized locally. The content is stunning, but the title is ambiguous and obscure, neither conveying the news content correctly, nor with thoughtfulness, instead, sensory stimulation and low taste, and only pursues click-through rate, yet neglect the social responsibility of media.

Headline is the "eyes" of the news, which largely determines the click-through rate and reading rate of the news. The headline of infotainment from Tanzi should not use uncertain, pornographic, violent, ridiculing and even insulting vocabulary to get sensational in order to sell more. When writing news headlines, the app needs to be accurate, clear, unambiguous, straight to the gist of the news. It can be popular but not vulgar, no sensitive words as irritating news points to spread low-taste and meaningless information to pander to some audience's curiosity. The infotainment apps, taking Tanzi as representative, fully satisfies the leisure and recreation needs of all levels of the audience, especially young people, extending the entertaining function of mass culture. However, as the producer and provider of information, Tanzi app must fulfill its responsibility as a cultural communicator and keep the bottom line of entertainment and moral bottom line of the media, to avoid the one-sided pursuit of the communication effect to lead the audience's blind pursuit of sensory stimulation.

\subsection{Pursue insightful report of infotainment and establish the correct entertainment values}

In 1972, McComb and Shaw verified agenda-setting function of the mass media, that is, certain topic or controversy, if it is emphasized by the mass media, the audience will consider it important and significant and it will cause the audience's response and changes on perception, attitude and behavior. Celebrities and stars, as public figures, their words and deeds, through the mass media's coverage, influence the audience's appreciation of beauty, behavior, moral pursuit and many other 
aspects like role models, and are easily accepted by the audience as the mainstream values and behavior, such as the modern audience's appreciation of body is taking thinness as beauty, which is exaggerated in the entertainment circle. From the statistics, it is found that Tanzi app propagates "thinness equals to beauty" too much, for instance, "Wanna be star? You must have a few clips behind your clothes", "Encounter Li Xiang family in Dubai, Wang Yuelun is so fat that regard as a nanny!”, “Chen Qiao-en is pregnant? Become so fat after several days not seeing her!”, these reports all subtly transmit the abnormal appreciation of beauty, i.e. "thinness equals to beauty". At the same time, stars as a high-income group, money-sucking capability and remuneration are the focus of the audience, and the reports of Tanzi app provide materialized values, like "Guan Xiaotong is so rich that her plastic bag is over 10 thousand!", "Li Xiang is so rich that she has every color of the hundred-thousand bag of Hermes", "Wang Sicong said only four words but earned eight million dollars", etc..

The mass media interpret news fact to the audience by constructing knowledge, forming norms and shaping consensus to affect the audience's perception and attitudes towards reality. Thus, the interpretation of hot entertaining event from the mass media is very important. The key points that Tanzi and other infotainment app need to focus are to enhance the depth of infotainment coverage, multi-dimensionally observe, analyze and review entertainment events and phenomenon. Tanzi app should be the reporter of the news rather than the manufacturer of the news, covering the entertainment event and people multi-dimensionally, in order to be close to different levels of audience's expectations of entertainment news and give full play to the power of idols. Entertainment celebrities are also the ordinary people and need to face the survival from various circumstances as well. Tanzi app should start from the celebrity's shinning point, from the audience's spiritual and cultural needs, make insightful and valuable entertainment reports to shape a healthy and complete value system from the audience's consumer intention, aesthetic taste, lifestyle and social attitudes and so on.

\section{Conclusion}

With the continuous development of society, the public requirement on entertainment increasingly improve. Infotainment app with its specialty, centrality and popularity quickly occupy the audience's vision. Generally, Chinese infotainment apps all take celebrity star as the main reporting elements, concern about the entertainment events and leading edge, and aims to expanding the coverage, integrating fashion, health, youth culture, local characteristics and other content into the reporting topic. They strive to avoiding homogenization in competition, attracting the audience with original review and transforming from simple sensory entertainment to the pursuit of cultural accomplishment and deep involvement of the topic. On the other hand, the reports of infotainment app are still using many buzzwords to create gimmicks, increasing click-through rate and attention by gossip, scandal, flirtatious, boring and ridiculing content, deliberate hype, title inconsistent with the content. Infotainment app, as a gathering area of entertainment news, except to meeting the audience relaxing and entertaining needs, should also meet the audience needs of the mass culture and popular culture. Only be virtuous and edutaining, and provide healthy entertainment for the audience, Chinese infotainment app can achieve their long-term development and give full play to the commonweal of the mass media.

\section{References}

[1] Pan Lu. Ethical Construction of Entertainment News [D]. Shanxi University, 2016.

[2] Ren Huizhen \& Cheng Lirong. Communication Strategies of Aggregating Information APP [J]. Press Circles, 2016, (05): 64-66.

[3] Zhang Yue. Between Happiness and Conscience [D]. Shaanxi Normal University, 2015.

[4] Zhang Li. Comparative Study on Network News Headlines and Newspaper News Headlines [D]. Shandong Normal University, 2015. 
[5] Chen Shi. The Relationship of Representation Communication Elements of Entertainment News on Weibo [J]. Media Observer, 2014, (07): 38-40.

[6] Gao Jie. The Value Orientation of Entertainment News under Pseudo-Environment [D]. Shaanxi Normal University, 2013. 